Hassan M.U., Akcamete-Gungor A., and Meral C. (2017). "Investigation of Terrestrial Laser Scanning Reflectance Intensity and RGB Distributions to Assist Construction Material Identification." In: LC3 2017: Volume I - Proceedings of the Joint Conference on Computing in Construction (JC3), July 4-7, 2017, Heraklion, Greece, pp. 507-515. DOI: https://doi.org/10.24928/JC3-2017/0312.

\title{
INVESTIGATION OF TERRESTRIAL LASER SCANNING REFLECTANCE INTENSITY AND RGB DISTRIBUTIONS TO ASSIST CONSTRUCTION MATERIAL IDENTIFICATION
}

\author{
Muhammad Usman Hassan ${ }^{1}$, Asli Akcamete-Gungor ${ }^{2}$, Cagla Meral ${ }^{3}$,
}

\begin{abstract}
Terrestrial Laser Scanning (TLS) allows collection of dense 3D point cloud data that captures a structure's as-is conditions. The geometric information from the collected data could be used to generate a 3D-model of the structure. However, the generated model usually lacks functional information - a basic requirement for a semantically rich information model. Some of the functional information (such as cost, mechanical and thermal performance) could be derived if the material used in a particular geometry is identified. The material related information in the collected data, which is mostly underutilized in the construction industry, could be potentially used for material identification. This paper investigates the possibility of material identification from the TLS data using the obtained scaled reflectance intensity $\left(\mathrm{I}_{\mathrm{i}}\right)$ from the laser scanner and the Red-Green-Blue (RGB) values from the in-built camera images. For this purpose, structural concrete, light-weight concrete and clay brick samples were scanned under two different conditions: (i) high/low ambient illumination, (ii) dry/wet surface. Obtained results show that scanned materials have different $I_{i}$ distributions. The illumination conditions have no effect on the obtained $\mathrm{I}_{\mathrm{i}}$ distribution; however, the surface moisture conditions vary the measured $\mathrm{I}_{\mathrm{i}}$ distributions considerably. Recorded RGB values of the illuminated samples could be used as a secondary parameter for material identification. These results show that TLS has a potential of augmenting the visual material identification processes.
\end{abstract}

Keywords: Laser scanning, point cloud, automated material identification, construction materials.

\section{INTRODUCTION}

Laser scanners are active sensors that emit coherent (in phase), monochromatic (single frequency) and highly directional near-infrared laser pulse which are used for contact free distance measurement to objects (Lemmens 2011; Kaasalainen et al. 2008). Terrestrial Laser Scanners (TLS), ground-based laser scanners mounted on a tripod, are used for capturing, at street view, the surface of objects in the surrounding, such as bridges, dams, building facades, trees or cultural heritage sites (Lemmens 2011).

Laser scanning is presently one of the most utilized 3D data acquisition methods, which can be used for structural health monitoring (Park et al. 2007), deformation measurement

1 Phd Candidate, Department of Civil Engineering, Middle East Technical University, Ankara, Turkey, usman.hassan@metu.edu.tr

2 Assistant Professor, Department of Civil Engineering, Middle East Technical University, Ankara, Turkey, akcamete@metu.edu.tr

3 Assistant Professor, Department of Civil Engineering, Middle East Technical University, Ankara, Turkey, cmeral@metu.edu.tr 
(Park et al. 2007; Lovas et al. 2008), and damage assessment (Olsen et al. 2010) in civil engineering structures. Topographical and survey related application is done using laser scanners to monitor geomorphic processes (Lim et al. 2005) and compute earthworks volume (Du and Teng 2007). Laser scans along with images (Liu et al. 2012) obtained at fixed intervals are combined to provide as-built documentation and material quantity information which can be utilized for progress monitoring (El-Omari and Moselhi 2008). Laser Scanning provides large amount of high resolution data in computationally expensive and costly manner, while cameras although cheap cannot provide laser scanner level accuracy when 3D information is required. Cameras may lack 3D data acquisition capabilities and are unable to generate point clouds at the rate of laser scanner but their ability to capture color information can be exploited for material identification and recognition related problems.

Construction materials are installed in various stages of projects and in various forms hence material identification is an important aspect from construction management perspective. Construction material identification is necessary for scope verification and progress monitoring purposes, which are performed by validating that the right type of material is installed at the right time in accordance with the schedule and specifications. Currently material identification is a manual and error prone process which involves visual identification of material. On built structures, the identification of materials is either done by visual means, core cutting, or highly specialized equipment, which can be unreliable, destructive, time consuming, and expensive. Material information can be attained from laser scanning data based on scaled reflectance intensity distributions, saving valuable time and resources. Automated construction material identification can create a record of material types and once correlated spatially, can populate as-built Building Information Models (BIM) with material information.

The goal is to come up with a robust method for material identification through laser scanning assisted with images, which can provide foundation for progress monitoring, scope verification, and quality control. For this purpose, in this paper, effects of reflecting surface properties on reflectance intensity and RGB distributions are investigated.

\subsection{Material Identification}

The laser beam reflectance intensity $\left(\mathrm{I}_{\mathrm{i}}\right)$ is the ratio of reflected to incident radiation from the scanned area in the laser wavelength (Song et al. 2002; Höfle \& Pfeifer 2007). Using $\mathrm{I}_{\mathbf{i}}$ from TLS as a parameter for material identification has been investigated in various research studies (Lichti and Harvey 2002). The inner crystalline structure of the material is determined in a non-invasive manner using TLS for material classification (Costantino \& Angelini 2013), showing that difference in intensity values is related to material composition. However, environmental parameters such as illumination, angle of reflectance, color of materials etc. also have an effect on $\mathrm{I}_{\mathrm{i}}$ (Voegtle et al. 2008; Reshetyuk 2006; Lee et al. 2010). TLS was used to determine amount of clay content of rock samples and it was observed that intensity data for marl, limestone and clay show difference in laboratory conditions and field settings (Franceschi et al. 2009). Limestone coal and red brick have shown different laser beam $\mathrm{I}_{\mathbf{i}}$ distributions (Lichti and Harvey 2002). Behaviour of construction materials like steel and wood was also observed (Voegtle et al. 2008) and laser beam $I_{i}$ mean values were found to be different. Even different types of wood had different laser beam reflectance intensity mean values and range.

In this paper, laser scanning using time-of-flight with wave Digitization technology is used which is different from time-of-flight based laser scanners used in previous research 
studies (Voegtle et al. 2008). The objective is to test this latest technology and augment laser scanning based material identification techniques that can be used for material identification and scope verification. To achieve this, material signatures for commonly used construction materials are identified. RGB values are provided along with reflectance intensities to act as additional parameters, in case obtained reflectance intensity distribution from laser scanning data is not unique to one material.

\section{EXPERIMENTAL METHODS AND DATA ANALYSIS}

Commonly used principles of taking measurements with TLS are phase-based and timeof-flight measurements (Lemmens 2011). Phase-based mechanism measures the phase of incident and reflected wave; and calculates the distance from the object based on the phase shift. The time-of-flight technology measures the time taken by a pulse to reflect back from an object and reach the scanner and depends upon nano second pulses, therefore creates a discrete stream. The phase shift method sends continuous stream of signals and therefore gives quicker results however maximum range is limited to the region in which unique ranges can be determined. Time-of-flight technique involves discrete signals which makes it slower.

Current research is conducted with Leica ScanStation P40 which uses ultra-high speed time-of-flight by waveform digitizing technology and emits a laser beam of $1550 \mathrm{~nm}$ wavelength with the scan rate of 1 million points per second. The full-waveform digitization is used to record the intensity time profile of the outgoing and returning signals which are used to determine unique features like peaks and centroids using digital signal processing. The time difference between selected features of incident and reflected waves give the distance of the object from the sensor (Fernandez-Diaz and Carter 2013).

Materials reflect certain wavelengths of light and absorb the rest and camera sensors capture light wave in visible spectrum reflected from the object. Leica P40 has a built in camera which contains a charge coupled device (CCD) containing photosensitive diodes that produce electric charge on pixels present on it, whenever light is incident upon them. The CCD is divided into a large number of light-sensitive areas known as pixels. The higher the luminosity of light, higher is the amount of charge accumulated on the pixel. The charge is transported to an array and read which can be used to build up an image. Visible spectrum lies between 400-700 nm. The wavelength of Red, Green and Blue colors are standardized at $700 \mathrm{~nm}, 546.1 \mathrm{~nm}$ and $435.8 \mathrm{~nm}$ respectively. The wavelength intensities are distributed between the spectral band on the basis of Grassman's law based on a set of rules to define Red, Blue and Green for a full visible spectrum (Mihai \& Străjescu 2007). The CCD sensor itself cannot differentiate between colors; therefore, color filters are utilized to determine amount of incident color (Nice and Gurevich 2012). Colour filters allows a very narrow spectral bandwidth corresponding to red, blue and green wavelengths of visible spectrum to pass through. Once red, blue and green wavelength intensities are obtained, they can be combined using RGB color model to represents true color visible to human eye. RGB color model is illustrated by Cartesian model with each color represented on each orthogonal axis. Each single point in the RGB subspace can define a particular color.

In this study, several scans were performed on commonly used materials in construction industry. These materials are installed and visible during the construction phase, until they are covered with cladding, insulation, or finishing. The materials were randomly selected from current inventory and no special specimen is prepared. Specifications of samples and post-processing areas of each scan are given in Table 1. 
Investigation of Terrestrial Laser Scanning Reflectance Intensity and RGB Distributions to Assist Construction Material Identification

Table 1: Sample Specifications and Scan Post-Processing Areas

\begin{tabular}{ccccc}
\hline Material & Composition and Color & $\begin{array}{c}\text { Planar Surface } \\
\text { Finish }\end{array}$ & $\begin{array}{c}\text { Large Area } \\
\mathbf{( \mathbf { c m } ^ { 2 } )}\end{array}$ & $\begin{array}{c}\text { Small Area } \\
\left(\mathbf{c m}^{2}\right)\end{array}$ \\
\hline $\begin{array}{c}\text { Concrete } \\
\begin{array}{c}\text { Light weight } \\
\text { concrete }\end{array}\end{array}$ & Homogeneous; grey & Smooth & 2018.9 & 343.7 \\
Clay brick & $\begin{array}{c}\text { Homogeneous; light grey } \\
\text { brownish orange }\end{array}$ & Porous & 532.0 & 133.0 \\
& Textured & 466.0 & 168.6 \\
\hline
\end{tabular}

Specimens were all kept upright throughout the test and were cleaned and dried before the test was performed to remove impurities. Figure 1 shows pictures of specimens used for the test.
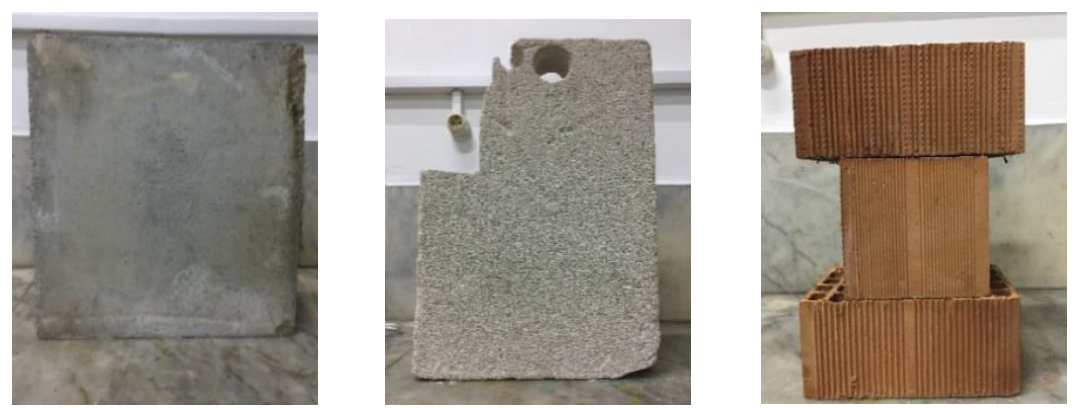

Figure 1: Materials (Left to Right); Concrete, Bricks, and Lightweight Concrete.

Concrete block was greyish in color with smooth surface and minor calcification on the peripheries. There were no reinforcements present inside the block. Light weight concrete was light grey in color. Its surface was dusty and uneven with small visible pores. Clay bricks were brownish orange in color. These bricks were stored at open-air conditions, and exposed to environmental elements for a long period of time.

In this study, scans were conducted in controlled lighting conditions with constant climatic conditions in a room with no windows and only artificial lighting. Specimens were placed $3 \mathrm{~m}$ away from the TLS. The specimens were placed at the same elevation as the scanner, in order to ensure perpendicularity of rays striking the specimen. To save time and memory, areas of interest were scanned by limiting horizontal and vertical rotation of the scanner. The sample were scanned under two different conditions: (i) high/low ambient illumination, (ii) dry/wet surface.

Cyclone software was used as the raw data parsing software for post-processing of the collected point cloud data and the images from native camera. During post-processing, anomaly (honey comb, calcification, and dust) free areas of the total scan were selected.

The specimen edges were also avoided due to greater noise at interfaces between different materials. Output from the software is given as $\left\{\left(\mathrm{x}_{\mathrm{i}}, \mathrm{y}_{\mathrm{i}}, \mathrm{z}_{\mathrm{i}}, \mathrm{I}_{\mathrm{i}}, \mathrm{R}_{\mathrm{i}}, \mathrm{G}_{\mathrm{i}}, \mathrm{B}_{\mathrm{i}}\right), \mathrm{i}=\right.$ $1,2,3, \ldots \ldots, N\}$ where $x_{i}, y_{i}$ and $z_{i}$ are the Cartesian coordinates; $I_{i}$ is the scaled reflectance intensity; and $R_{i}, G_{i}, B_{i}$ are the 8-bit integer (from 0 to 255) RGB-values for the $i^{\text {th }}$ acquired point. Based on the intensity characteristics (minimum, maximum etc.) of the scanner, the software linearly scales the measured reflectance intensity value between 0 and 1 to obtain $I_{i}$. The output of the software is exported for further data analysis. In the data analysis software, first the $R_{i}, G_{i}$ and $B_{i}$ values are linearly scaled between 0 to 1 . 
Then, for each scan $I_{i}, R_{i}, G_{i}$ and $B_{i}$ distributions of the selected scan area are represented as histograms.

\section{RESUlTS AND DisCUSSIONS}

\section{1 $\mathbf{I}_{\mathbf{i}}$ distribution}

The $\mathrm{I}_{\mathbf{i}}$ distribution of each material varies according to its capacity to absorb and reflect incident laser beam which depends on intermolecular distance and surface properties. The differences in the $I_{i}$ distributions of the scanned surfaces can be used for material identification. Figure 2 shows obtained $\mathrm{I}_{\mathrm{i}}$ distributions of large post-processing areas (LA) on each sample. A different $I_{i}$ distribution has been observed for each scanned material.

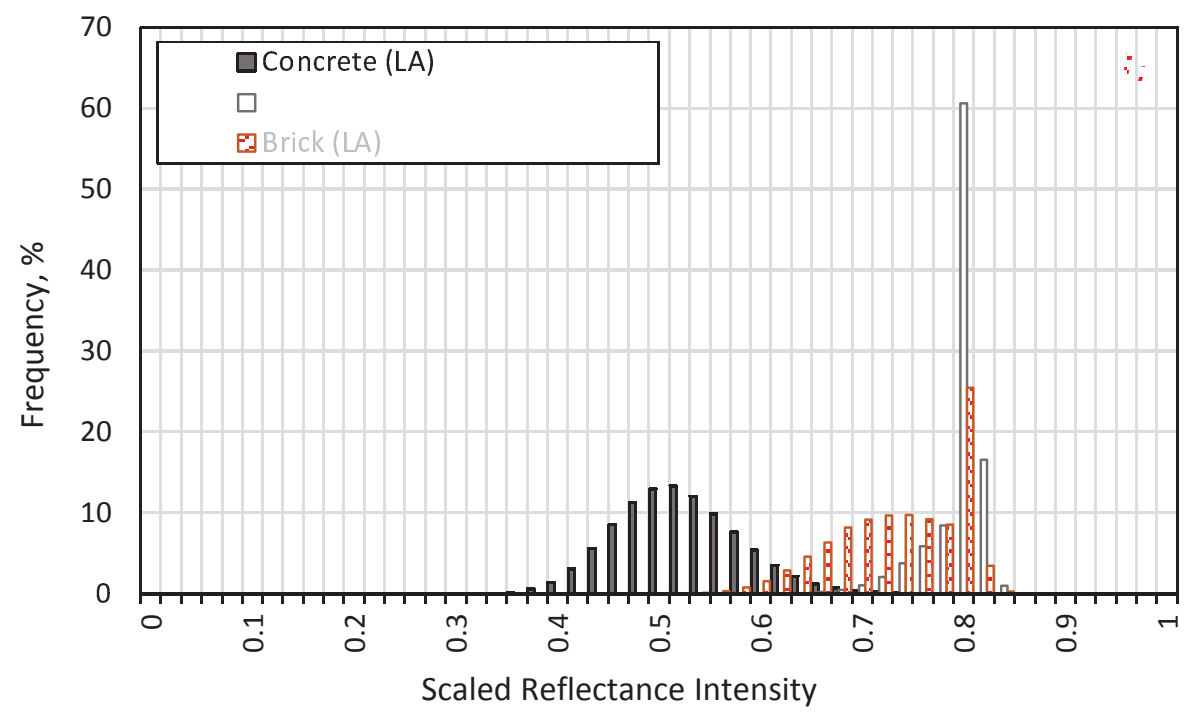

Figure 2: $\mathrm{I}_{\mathrm{i}}$ distributions of large post-processing areas (LA) on concrete, light weight concrete and clay brick specimens.

The material specific $I_{i}$ distributions need to be repeatable for scans taken from different parts of a specimen given that the measurement conditions are kept the same. For each scan, the precision of the $\mathrm{I}_{\mathbf{i}}$ distribution was checked by comparing $\mathrm{I}_{\mathrm{i}}$ histograms obtained for a LA and a small, randomly selected post-processing area (SA). Representative LA and SA are shown in Figure 3a. Figure 3b-d shows comparison of LA and SA intensity distributions of each material. Obtained distributions from the smaller areas were similar to those obtained from the larger areas suggesting that even small, randomly selected areas of the scan can be representative of the complete intensity distribution. 


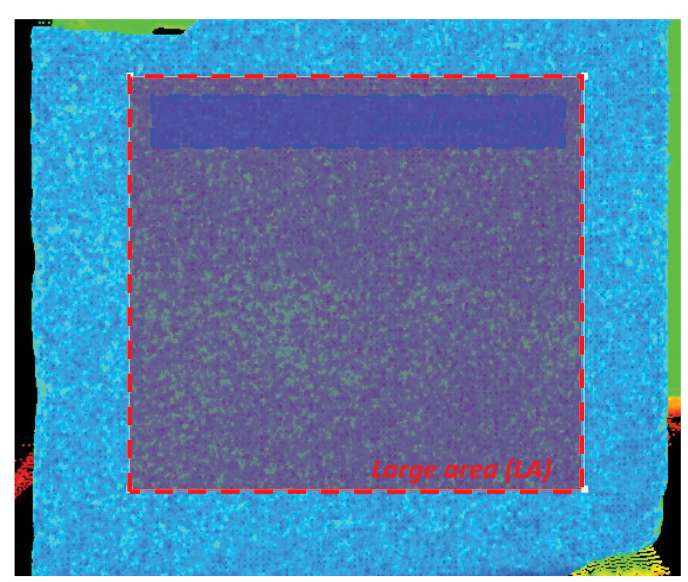

(a)

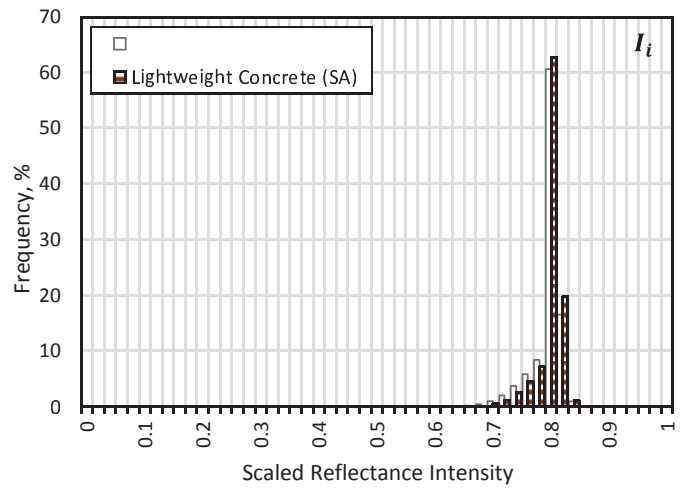

(c)

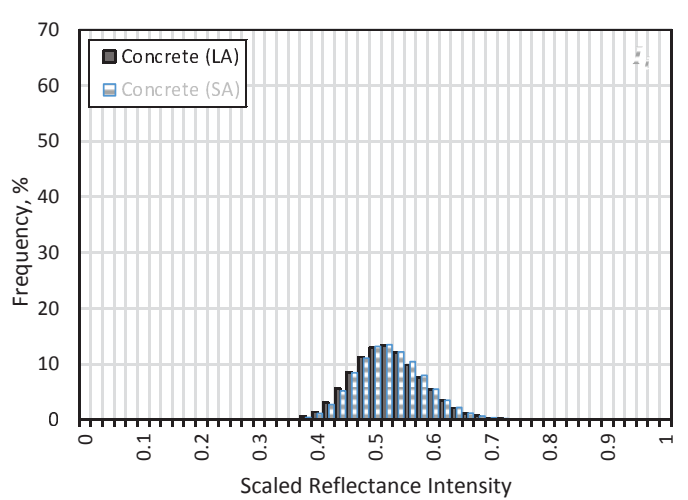

(b)

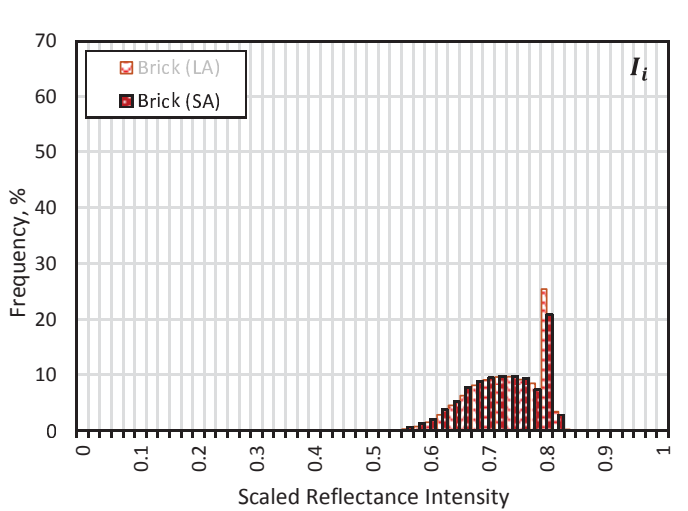

(d)

Figure 3: (a) Representative small (SA) and large (LA) post-processing areas; Comparison of $\mathrm{I}_{\mathrm{i}}$ distributions of SA and LA of (b) concrete, (c) light-weight concrete, and (d) clay brick.

The $\mathrm{I}_{\mathrm{i}}$ distributions of samples at high and low ambient illumination are given in Figure 4a. The results at different illumination levels showed no significant difference than the results presented at Figure 2. Laser scanning is an active visualization technique. A laser scanner has its own energy source producing electromagnetic laser beam, unlike passive imaging which is critically dependent on lighting conditions (Shengyong Chen 2008). The laser beam being monochromatic and coherent, is filtered from all other radiations incident on the detector. Laser beams travel at speed of light and presence or absence of light doesn't affect their movement through a medium or reflectance from a surface. As long as a scanner uses only one wavelength to collect the point cloud data, the $I_{i}$ of a scanned point remains unaltered.

$I_{i}$ values are greatly affected by humidity especially for scanners whose wavelength is in the near infrared range, which corresponds to strong water related absorption (Franceschi et al. 2009). The $\mathrm{I}_{\mathrm{i}}$ distributions of the scans on high surface moisture specimens are given in Figure 4b. The results shifted towards left when compared to the results presented at Figure 2 which suggests that the surface moisture state can be predicted from the $I_{i}$ distributions. The moisture condition of a surface effects the $I_{i}$ distributions. The laser beam interacts with the surface between the material particles and the film of water coating them following Fresnel Reflection. A portion of the incident laser beam is reflected from the water surface, while some is transmitted through the water and reflected from the material particles beneath. The remaining fraction is absorbed by water. 
The total internal reflection at the interface between thin film of liquid and air increases the probability of energy absorption (Lekner \& Dorf 1988). A higher absorption rate suggests lower reflectance characteristic of a high surface moisture specimen (Philpot 2010).

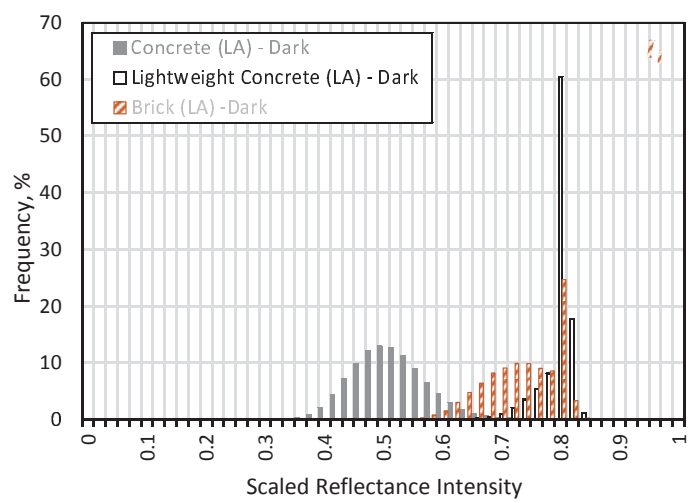

(a)

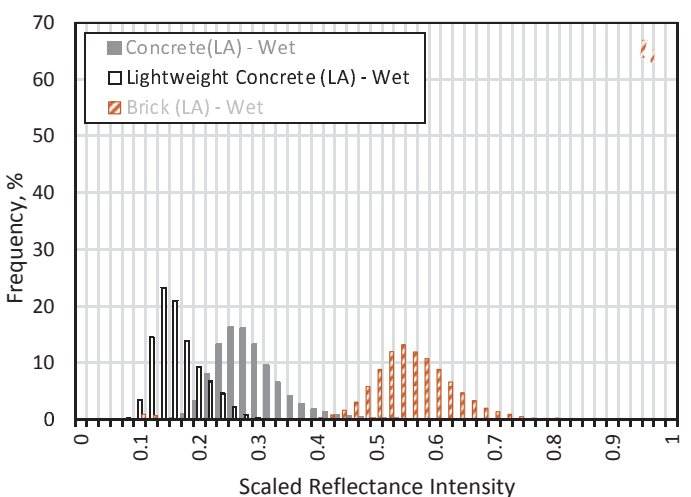

(b)

Figure 4: $I_{i}$ distributions of concrete, lightweight concrete and clay brick samples at (a) low ambient illumination and (b) high surface moisture.

\subsection{Visible spectrum}

Histograms of the scaled $R_{i}, G_{i}$ and $B_{i}$ values were plotted in Figure 5. Concrete and lightweight concrete both are in a shade of grey; therefore, their RGB histograms broadly overlap even though they have different peaks, means and standard deviations. The RGB histograms of the clay brick, which is in a shade of orange, is considerably different than those of the concrete and light-weight concrete in green and blue bands. The distribution of RGB values can assist $\mathbf{I}_{\mathbf{i}}$ distribution in material identification by augmenting material specific information. However, material color information is dependent on various factors such as sensor type, temperature, and lighting conditions. Due to large number of variables involved, it is challenging to solely depend on RGB values for material identification.

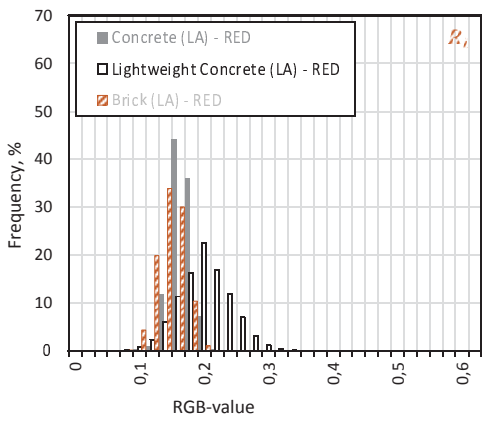

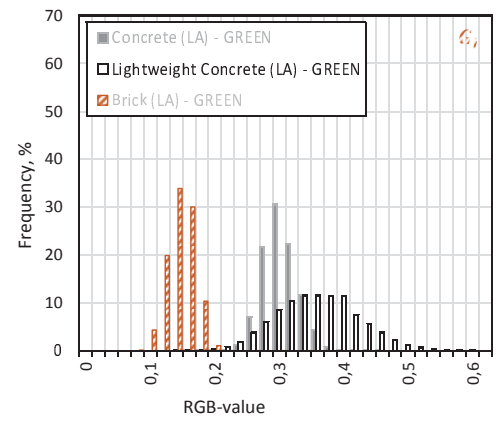

(b)

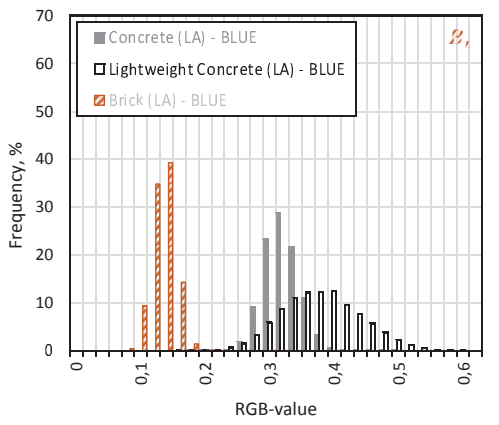

(c) brick samples at (a) Red band, (b) Green band and (c) Blue band.

\section{CONCLUSIONS AND FUTURE WORK}

In this study, a concrete, a clay brick and a lightweight concrete block specimen were scanned through a terrestrial laser scanner in order to observe the effects of reflecting surface properties on $\mathbf{I}_{\mathbf{i}}$. The experiments showed that each material scanned has a unique 
$\mathrm{I}_{\mathrm{i}}$ distribution when defect free areas were scanned, proving relevance of laser scanning data for material identification. Low and high ambient illumination does not have an effect on $\mathrm{I}_{\mathrm{i}}$ distributions because of active visualization mechanism of laser scanning. Presence of moisture on material surface increase absorption of incident laser beam causing decrease in reflection and shifting $\mathrm{I}_{\mathrm{i}}$ distributions towards the lower end of laser beam reflectance intensity range.

In the visible spectrum, the RGB values for brick was considerably different than concrete and light weight concrete. Being greyish in color, RGB values of concrete and light weight concrete did overlap, but their mean, range and standard deviation was dissimilar. The RGB intensity histograms will act as additional parameters to reduce error in material identification by augmenting results obtained from laser beam reflectance intensity.

The results can be further refined by experimenting in different natural lighting conditions, since all experiment were carried out in artificial lighting. All measurements were also taken from fixed distance of $3 \mathrm{~m}$ and effect of distance on intensity values was not observed. Different specimens having same characteristics should be scanned to observe variation of intensity values in between specimens of same material type. The scans need to be performed on an actual construction site in varying climatic conditions to observe the deviation in $\mathrm{I}_{\mathrm{i}}$ values. The native P40 camera has 4 Mega-pixel resolution and results were not of high quality. In order to offset effect of sensor and lighting conditions, HSI color model can be evaluated instead of RGB color model, on images taken from a professional DSLR camera.

\section{REFERENCES}

Costantino, D. \& Angelini, M.G., 2013. Qualitative and Quantitative Evaluation of the Luminance of Laser Scanner Radiation for the Classification of Materials. ISPRS International Archives of the Photogrammetry, Remote Sensing and Spatial Information Sciences, XL-5/W2(September), pp.207-212.

Du, J.-C. \& Teng, H.-C., 2007. 3D laser scanning and GPS technology for landslide earthwork volume estimation. Automation in Construction, 16(5), pp.657-663.

El-Omari, S. \& Moselhi, O., 2008. Integrating 3D laser scanning and photogrammetry for progress measurement of construction work. Automation in Construction, 18(1), pp.1-9.

Fernandez-Diaz, J.C. \& Carter, W.E., 2013. Understanding Waveform Digitizing and Waveform Data Processing,

Franceschi, M. et al., 2009. Discrimination between marls and limestones using intensity data from terrestrial laser scanner. ISPRS Journal of Photogrammetry and Remote Sensing, 64(6), pp.522-528.

Höfle, B. \& Pfeifer, N., 2007. Correction of laser scanning intensity data: Data and model-driven approaches. ISPRS Journal of Photogrammetry and Remote Sensing, 62(6), pp.415-433.

Kaasalainen, S. et al., 2008. Brightness measurements and calibration with airborne and terrestrial laser scanners. IEEE Transactions on Geoscience and Remote Sensing, 46(2), pp.528-533.

Lee, I.S. Lee, J.O., Park, H.J., Bae, K.H., 2010. Investigations into the influence of object characteristics on the quality of terrestrial laser scanner data. KSCE Journal of Civil Engineering, 14(6), pp.905-913. 
Lekner, J. \& Dorf, M.C., 1988. Why some things are darker when wet. Applied Optics, Vol. 27(7), pp.1278-1280.

Lemmens, M., 2011. Geo-information: technologies, applications and the environment, Dordrecht: Springer.

Lichti, D.D. \& Harvey, B.., 2002. the Effects of Reflecting Surface Material Properties on Time-of-Flight Laser Scanner Measurements. Geospatial Theory, Processing and Applications, (2001).

Lim, M. et al., 2005. Combined digital photogrammetry and time-of-flight laser scanning for monitoring cliff evolution. Photogrammetric Record, 20(110), pp.109-129.

Liu, X., Eybpoosh, M. \& Akinci, B., 2012. Developing As-built Building Information Model Using Construction Process History Captured by a Laser Scanner and a Camera. In Construction Research Congress. pp. 1232-1241.

Lovas, T. et al., 2008. Terrestrial laser scanning in deformation measurement of structures. In The International Archives of the Photogrammetry, Remote Sensing and Spatial Information Sciences. pp. 527-532.

Mihai, D. \& Străjescu, E., 2007. From wavelength to R.G.B filter. U.P.B. Scientific Bulletin, Series D, 69(2).

Nice, K. \& Gurevich, G.J., 2012. How Digital Cameras Work Understanding the Basics A Filmless Camera.

Olsen, M.J.,Asce, M., Kuester, F., Chang, B.J., Asce, S.M., Hutchinson, T.C., 2010.

Terrestrial Laser Scanning-Based Structural Damage Assessment. Journal of Computing in Civil Engineering, 24(3), pp.264-272.

Park, H.S., Lee, H.M., Adeli, H., 2007. A new approach for health monitoring of structures: Terrestrial laser scanning. Computer-Aided Civil and Infrastructure Engineering, 22(1), pp.19-30.

Reshetyuk, Y., 2006. Investigation of the Influence of Surface Reflectance on the Measurementswith the Terrestrial Laser Scanner Leica HDS 3000. Zeitschrift für Vermessungswesen, 2, pp.96-103.

Shengyong C., 2008. Active Sensor Planning for Multiview Vision Tasks, Springer Berlin Heidelberg.

Song, J.H., Han, S.H., Yu, K.Y., Kim, Y.I., 2002. Assessing the possibility of land-cover classification using lidar intensity data. International Archives of Photogrammetry, Remote Sensing and Spatial Information Sciences, 34, pp.259-263.

Voegtle, T., Schwab, I. \& Landes, T., 2008. Influences of different materials on the measurements of a terrestrial laser scanner (TLS). Proc. of the XXI Congress, The International Society for Photogrammetry and Remote Sensing, ISPRS2008, 37(V), pp.1061-1066. 